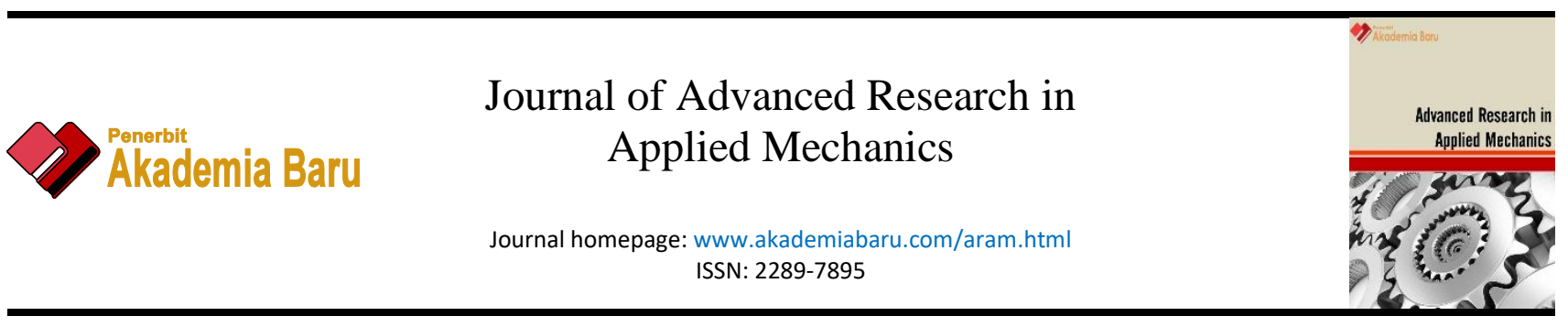

\title{
File and PC-Based CNC Controller using Integrated Interface System $\left(I^{2} S\right)$
}

\author{
Tengku Mohd Sharir Tengku Sulaiman ${ }^{1}$, Saiful Bahri Mohamed ${ }^{1,}{ }^{*}$, Mohamad Minhat ${ }^{2}$, Ahmad \\ Syafiq Mohamed², Ahmad Ridhuan Mohamed ${ }^{3}$, Siti Nurul Akmal Yusof ${ }^{4}$

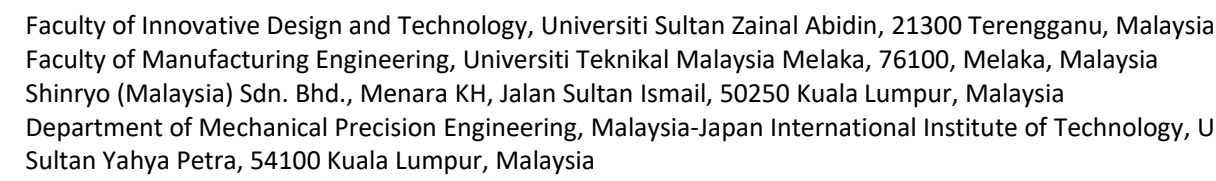

\section{ABSTRACT}

STEP file is known as a CAD neutral file which stands for Standard for the Exchange of Product model data for the next generation of data model between CAD/CAM and CNC system. It contains three-dimensional data in ISO 10303-21 format which ultimately all CNC programs can recognize. Now however, not all documents in the STEP file are appropriate for programming CNC G-Code. This paper explicates the design, development and testing of an Integrated Interface System (I²S) using knowledge discovery approach. The objective of this study is to provide helps for understanding the STEP-NC machining operation protocol. The system provides function of reading and extracting applicable data related with the machining functions from STEP file, that eventually write file format Geometrical Code (G-Code - ISO 6983) as an output file. G-Code is known as one of the low-level programming languages in CNC system. The sample of machined block designs from 3D CAD modeler where the features need to be machined from a blank workpiece and saved in the STEP neutral file format. In this work, the simulation in the Mach3 software and physical machining using 3-axis CNC milling machine installed with PC-based CNC controller has been validated.

Keywords:

STEP; STEP-NC; CNC; G-code; Graphic

User Interface

Copyright @ 2020 PENERBIT AKADEMIA BARU - All rights reserved

\section{Introduction}

In 1952, the Massachusetts Institute of Technology (MIT) was the first pioneer demonstrating the numerical control (NC) machine tool. NC started when the manually operated machine transformed into the automatic form of machine tools that first introduced principles of abstractly programmable logic, and today it continues with the incorporation of computer technology into the idea of numerical control leading to what we now know as computer numerical control (CNC). CNC is a cutting-edge idea in the manufacturing and machining industries. Such production machines relied on existing tools, which are equipped with motors designed to move and control the axes of the machine. CNC machine is managing the language of low-level programming known as $\mathrm{G}$ and M-codes

\footnotetext{
* Corresponding author.

E-mail address: saifulbahri@unisza.edu.my
}

https://doi.org/10.37934/aram.70.1.18 
(ISO 6983). G and M-codes are the basic building block of CNC machines [1]. The low-level G and Mcodes contains a very limited amount of information or data regarding the machine capabilities and the work piece characteristic which results in no information available on manufacturing process and machining functions [2-3]. G and M-code serves as a language of the computer between controller and machine itself. All the movements the system makes are guided using $\mathrm{G}$ and $\mathrm{M}$-codes. Ultimately $\mathrm{G}$ and $\mathrm{M}$-codes worked using the coordinates and points that ultimately led to the creation of paths for the devices. The $\mathrm{G}$ and $\mathrm{M}$-code language is designed in the era when the paper tape is the medium for moving data between computers and CNC systems. Nowadays the $\mathrm{G}$ and $\mathrm{M}$-code programs are generated using the computer aided manufacturing (CAM) tools by using the geometrical design data from computer aided design (CAD) tools as the input [4].

According to the machine brands and the manufacturing business enterprise there are many kinds of $G$ and $M$-code structure and they have their own unique $G$ and $M$-code format. This type of proprietary environment will result in incompatibility between the data format which limits further improvement of CNC-based machining [5]. CAD/CAM software program is used for designing and operating machineries, however the machines themselves require a final process. The process is known as the post-processing process, which guarantees that the code transmitted to them is readable in order to optimize the operation of the machine. Based on the CAD model, the CAM software will analyse and determine the type of tooling and create the tool paths based on the desired features. Therefore, to be able to do so, this process requires a CAM post-processor that produces the exact $\mathrm{G}$ and $\mathrm{M}$-code dialect used by the machine that is being targeted. For the CNC's case, it limited only for the programs within their own versions of $G$ and $M$-code. Apart from that, the shortcoming for the conventional NC programming are limited NC-programming interfaces, no connection to CAD data and technology, lack of shop-floor knowledge, lack of control and machine tools, and the need for a post-processor interface [6].

Surprisingly, with the rapid development of information technology associated with the CNC technology, a new approach of manufacturing system has been introduced, known as DABA (DesignAnywhere-Build-Anywhere) e-manufacturing system. The smooth data flow in the CAD-CAM-CNC chain is a very important aspect of the process in order to make e-manufacturing a success. The STEP (ISO 10303: Standard for the Exchange of the Product data) developed since the 1980s is the data standard for exchanging product data between CAD/CAM systems. To overcome the limitations of data flow among CAD/CAM to CNC [7-8], the STEP data structure has been introduced. The STEP data structure will provide a new data exchange interface between the CAD/CAM/CNC by using the term of open architecture environment.

Open architecture environment software which allows the bidirectional data flow from starting from the design stage until the machining stage. Then, the data flow is saved in a STEP data file using Part 21 data file. Part 21 data file is an interpretation from the solid model that contains relevant information and represent the information in an internal format that allows simple editing and viewing from the file of Part 21 itself [9-11]. STEP file is an international standard which consisting of the design features of the CAD model, which provides general support for the sharing of the data model in a standard CAD form between the CAD system or the software [12]. STEP is a standardized data structure that can be accessed, edited and stored only in a text format in any open source software. By using the STEP data structure, the post processor will no longer be used because the mechanism will bypass the post processor. This is because the information in the data structure file is sufficient to perform the machining without the need for a post processor.

The most breakthrough in the CNC field is that these STEP files bypass the post-processor and save more time for the manufacturing processes. 


\section{STEP Data Structure}

STEP promotes representation and exchange between all phases of the product life cycle, for example design, prototyping, process planning and production. ISO 10303 defines as a new alternative or language for the manufacturing industry, in particular for product development.

STEP can optimize the product data exchange and also provides a reference model for a complete description of product data over the entire product life cycle [13]. STEP is a high-level programming language, which contains the solid model features creating by using CAD software. It allows bidirectional data flow between CAD/CAM and CNC without any redundancy and loss of information [14]. In the bi-directional data flow, the exchange data should be defined using the object-oriented modeling method and no data leakages are allowed on CAD/CAM/CNC data chain.

Nowadays the new generation of intelligent controllers can interpret STEP file information to generate, simulate and optimize machining toolpaths [15]. STEP file is a universal data structure which means it is an open source data structure and not proprietary to any specific machine or brand and can be used on various open source machine tool controllers in the market. By using this STEP data file as the input file, the post-processor is not needed anymore because the information within the file is enough to generate $\mathrm{G}$-code and thus execute machining.

File extension for STEP file is STEP or STP file is an open source file and can be accessed using any standard software like Microsoft Word, Notepad, etcetera. STEP data structure consists of HEADER and DATA section. General information is described in HEADER section. This includes file title, author and schema information. In DATA section, the instances consist of design feature of the solid model, which is the Cartesian coordinate, Cartesian point, lines, axis, units, and so on. By having all information in the same file all changes are effective thus eliminating the need of post-processor. The STEP file is a rich file in term of solid features information and that is why the STEP file alone can be used to execute machining by generating $\mathrm{G}$ and $\mathrm{M}$-code. This is why STEP standards appear as the relevant solution to extend the interoperability of CAx chain to the CNC level.

\section{Integrated STEP/G-code Interface}

The integrated STEP/G-code interface is designed as the Graphical User Interface (GUI) between the STEP file and G-code in order to generate G-code based on the STEP file as the input file. Within the integrated interface environment, the programming algorithms are designed in such a way to perform reading the input file, processing, and writing the out-put file as presented in Figure 1.

The read module performs read the input file line by line and prepare that file reachable for further processing. Whilst, the processing algorithms tasks consists of three sub-module performing extraction/elimination, the processing and integration activities. This process used extraction rule to identify the geometric data and write it into a temporary file. There may be some duplication of data exist however, it will be eliminated using elimination rule. Further, the application of processing rule for the extracted data which is involving determination of working plane and $z$ axis, determination of work piece boundary, determination of feature boundary and determination of cutter movements will be carried out.

After determining and verifying all the process and required information, the integration process will then generate the tool path along with the required cutting parameters. Finally, the process of generating G-Code through the transformation module, transforming the processed integration data into a sequence of axis movement and tool functions as defined in ISO 6983. All these processes are carried out in the coordination layer and the conceptual system architecture of $\mathrm{I}^{2} \mathrm{~S}$ is shown in Figure 2. 


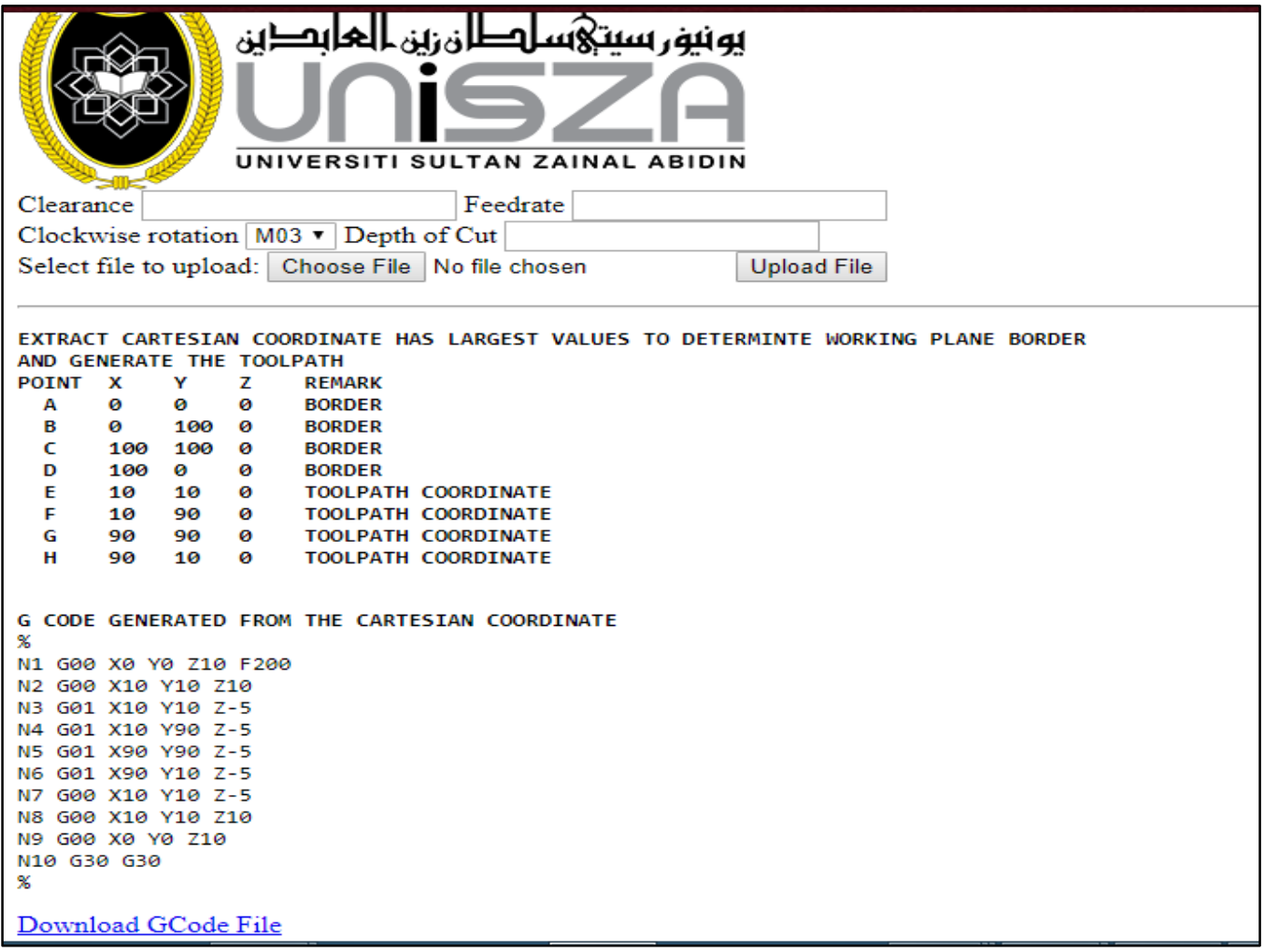

Fig. 1. Integrated STEP/G-code interface

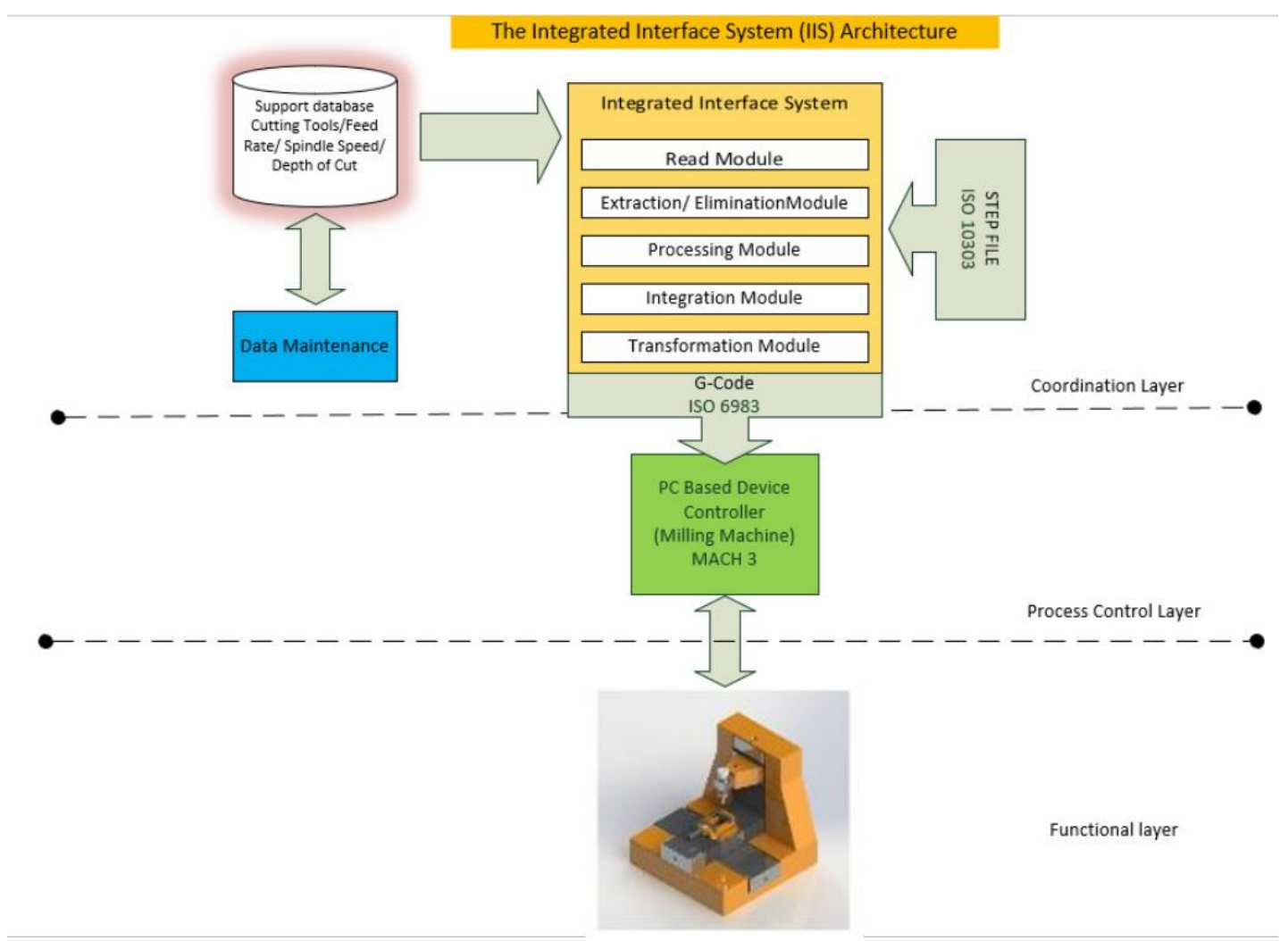

Fig. 2. The integrated interface system $\left(I^{2} S\right)$ architecture 


\section{STEP File and Solid Model}

The input file for this project uses only Part 21 STEP file instead of total STEP-NC file, because the cartesian coordinates are used to generate $G$ and $M$-code. A sample of 3D solid model has been designed and properly given dimension to represent straight line linear motion of G01 linear interpolation using the Solid work software as shown in Figure 3. This solid model is then saved in a STEP file. On the other hand, the solid model that is in SLDPRT file will be opened in CAM in order to simulate and generate the $G$ and $M$-code. The $x-y-z$ coordinate from the auto-generated $G$ and $M-$ code is compared with the cartesian coordinates in the STEP file that was previously saved for error check.

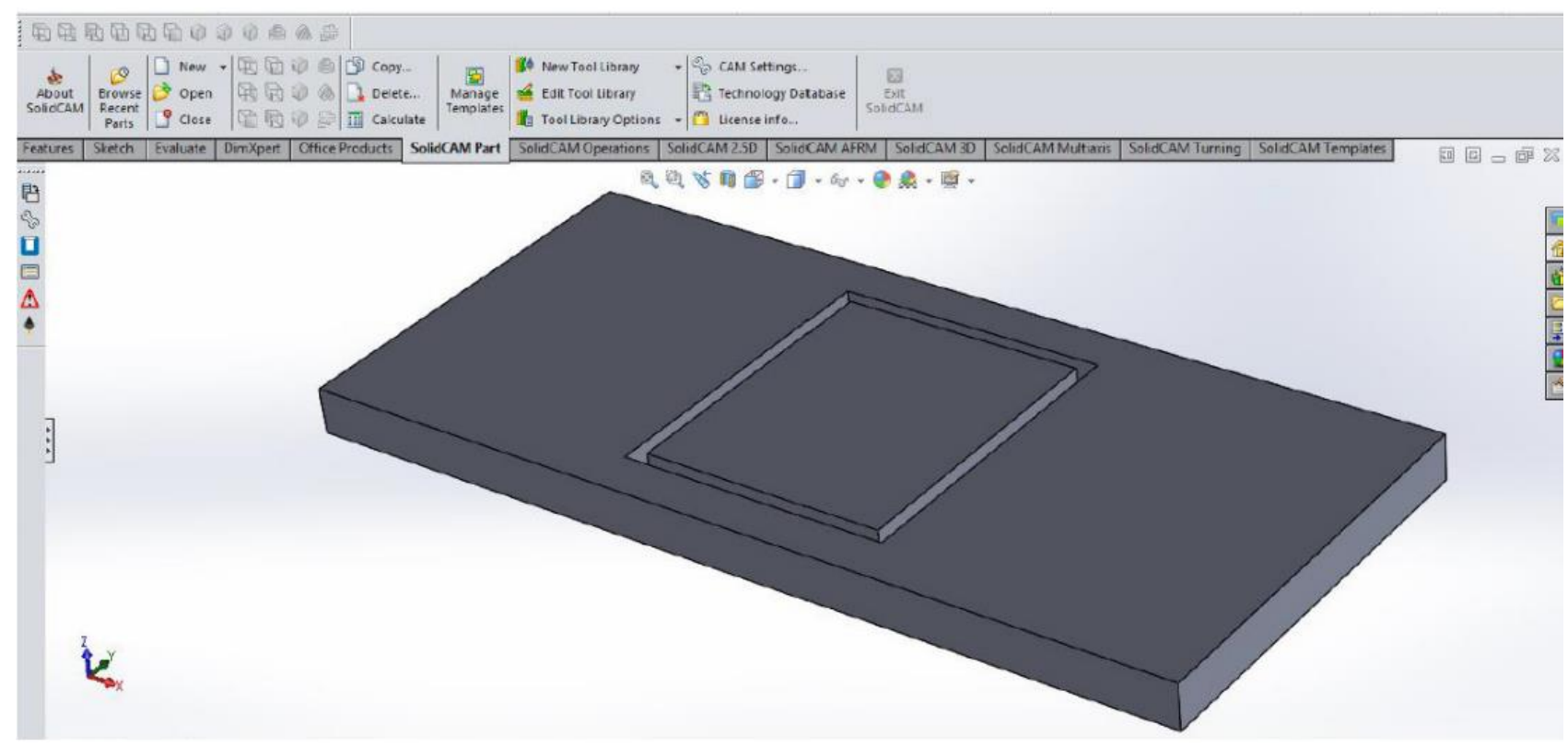

Fig. 3. Solid model in solid work design interface

\section{Machining Simulation and Testing}

In order to ensure the generated $\mathrm{G}$ and $\mathrm{M}$-code from the $\mathrm{I}^{2} \mathrm{~S}$ can be used prior to physical machining; the supplementary run is performed using the Mach 3 PC-based CNC controller according to the required setting parameters. The $\mathrm{G}$ and $\mathrm{M}$-code shall be able to generate the tool path in as shown in Figure 3, based on the required solid CAD design model. The tool path generated from the tool's movement is based on the $\mathrm{G}$ and $\mathrm{M}$-code that is created from the cartesian coordinates in the STEP file itself. This machining process involves the operation of slot milling using the square shaped end milling tool. The milling shape is just a simple island shape with the rectangle having depth of 5 $\mathrm{mm}$, which means that the $\mathrm{z}$ level will be -5 . The result from the simulation is shown in Figure 4 .

\section{Validation of Physical Machining}

The 3-axis CNC milling machine is used for the physical machining testing to validate the $\mathrm{G}$ and M-codes used earlier during the simulation. A piece of wood with dimension of $100 \mathrm{~mm} \times 200 \mathrm{~mm} \times$ $20 \mathrm{~mm}$ is used as the work piece for the machining purpose. The machining operation took place on a 3-axis CNC milling machine equipped with Mach 3 is open source CNC controller as shown in Figure 
5. It can be seen from the figure that the $x-y-z$ coordinate from the auto-generated $\mathrm{G}$ and $\mathrm{M}$-code has a good agreement with the cartesian coordinates in the STEP file.

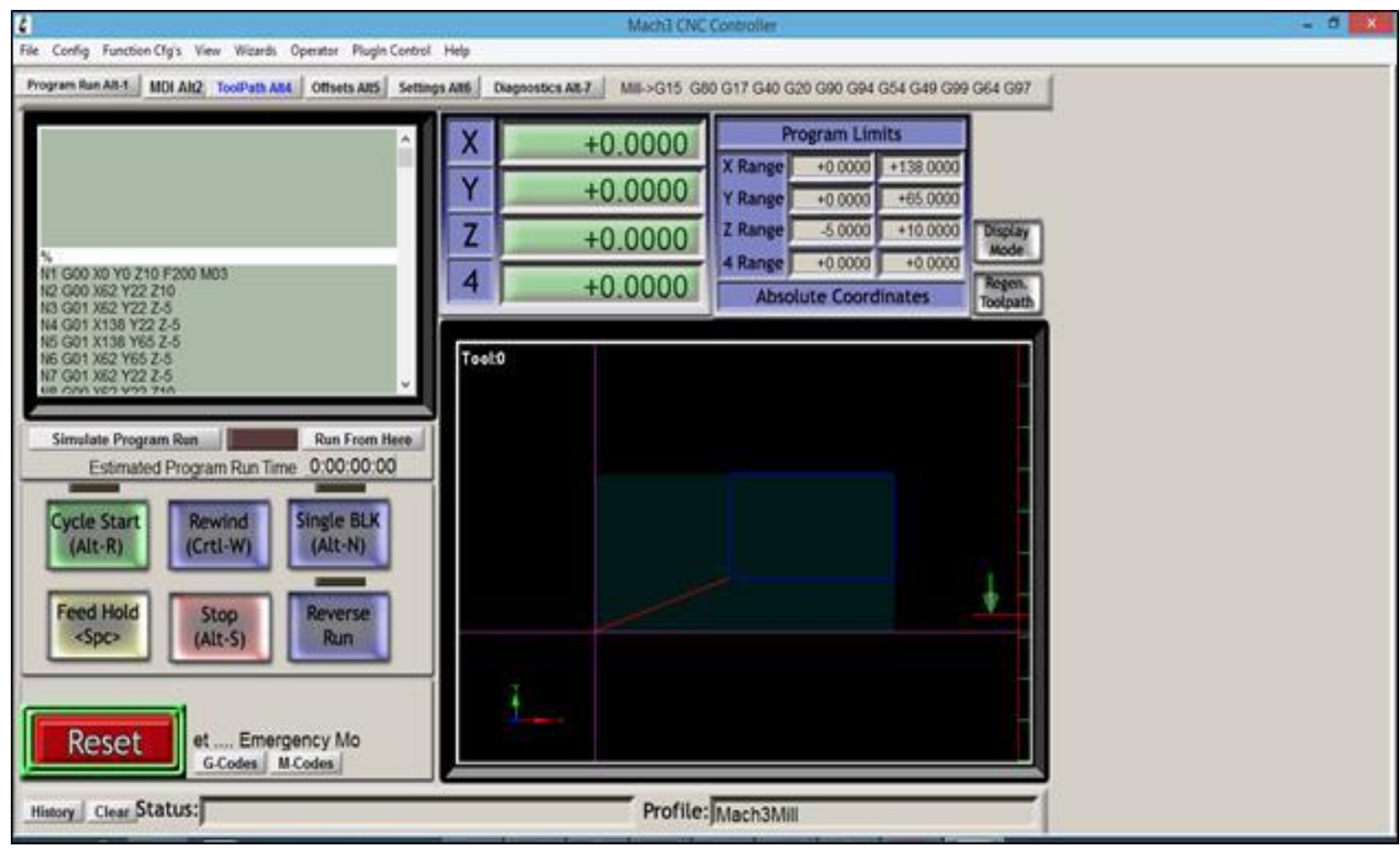

Fig. 4. Mach 3 simulation result

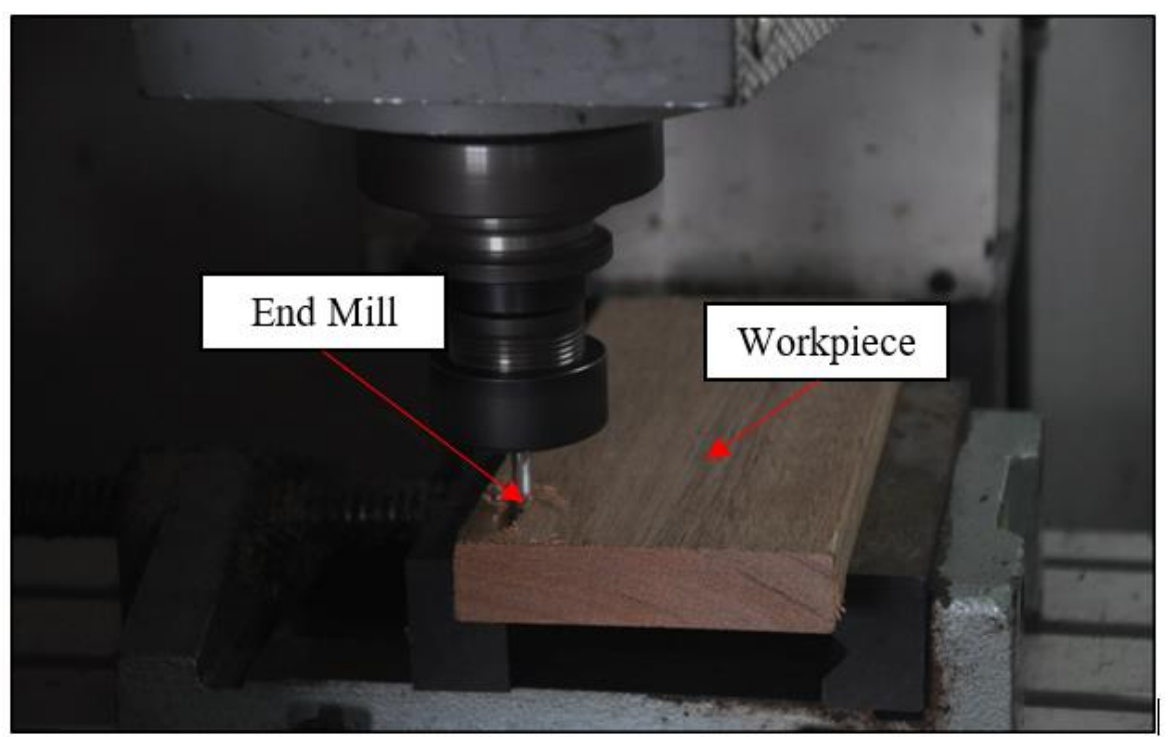

Fig. 5. 3-Axis CNC milling machine in operation

\section{Conclusions}

Developing the integrated STEP/G-code interface successfully eliminates the need for postprocessor since machine-specific information is not required. Additionally, $I^{2} S$ system developed successfully generates the $\mathrm{G}$ and $\mathrm{M}$-code based on the information in the STEP file. By using the cartesian coordinates in the STEP file, it can generate the $\mathrm{G}$ and $\mathrm{M}$-code based on the solid CAD design that is required. This is because the $G$ and $M$-code consists only of coordinates and certain parameters for machining strategies. For CNC machining, this process is a read-write-read-write 
(RW/RW) process. Initially, when the solid model is saved as the STEP neutral file format, the software reads and saves all the solid features as the required file. Then, the write process, is when the information within the solid model is being summarized as a text file format which contains all the design features for a particular model. Later, the read process again, is when the extraction of the cartesian coordinates in the STEP file and finally, the last write process, is when the generation of the $\mathrm{G}$ and $\mathrm{M}$-code based on the cartesian coordinates. Extraction, elimination, processing, and integration of the cartesian coordinates being done automatically based on the STEP file fed as an input file.

For the future recommendations, the square solid CAD model representing a linear motion interpolation $\mathrm{G} 01$ can be changed into circular interpolation motion G02. Of course, the algorithm involving generation of G-code for more complex tool path from STEP file would be a greater challenge.

\section{Acknowledgement}

The authors thank to the Universiti Sultan Zainal Abidin (UniSZA), Universiti Teknikal Malaysia Melaka (UTeM), Shinryo (Malaysia) Sdn. Bhd, Institut Teknologi Petroleum PETRONAS and the Ministry of Higher Education Malaysia for financial and general support of this research through the Fundamental Research Grant Scheme (Grant No. FRGS/1/2018/TK03/UNISZA/02/1).

\section{References}

[1] Mohd Asif Hasan. "Computer numerical control machines: An account of programming methods and techniques." Journal of Material Science and Mechanical Engineering 2 (2015): 14-17.

[2] Galanis, N. I., S. L. Afsaridis, S. N. Vlachostathopoulos, and D. E. Manolakos. "Manufacturing process with the use of protocol STEP-NC." In 3 rd ICMEN Conference, pp. 1-3. 2008.

[3] Živanović, Saša T., and Goran V. Vasilić. "A new CNC programming method using STEP-NC protocol." FME Transactions 45, no. 1 (2017): 149-158.

https://doi.org/10.5937/fmet1701149Z

[4] Kumar, Sanjeev, Aydin Nassehi, Stephen T. Newman, Richard D. Allen, and Manoj K. Tiwari. "Process control in CNC manufacturing for discrete components: A STEP-NC compliant framework." Robotics and Computer-Integrated Manufacturing 23, no. 6 (2007): 667-676.

https://doi.org/10.1016/j.rcim.2007.02.015

[5] Dubovska, Rozmarina, Jaroslav Jambor, and Jozef Majerik. "Implementation of CAD/CAM system CATIA V5 in Simulation of CNC Machining Process." Procedia Engineering 69 (2014): 638-645.

https://doi.org/10.1016/j.proeng.2014.03.037

[6] Minhat, Mohamad, Valeriy Vyatkin, Xun Xu, Soon Wong, and Zina Al-Bayaa. "A novel open CNC architecture based on STEP-NC data model and IEC 61499 function blocks." Robotics and Computer-Integrated Manufacturing 25, no. 3 (2009): 560-569. https://doi.org/10.1016/i.rcim.2008.03.021

[7] Othman, M. A., M. Minhat, and Z. Jamaludin. "An overview on STEP-NC compliant controller development." In IOP conference series: materials science and engineering, vol. 257, no. 1, p. 012048. IOP Publishing, 2017. https://doi.org/10.1088/1757-899X/257/1/012048

[8] Xiao, Wenlei, Lianyu Zheng, Ji Huan, and Pei Lei. "A complete CAD/CAM/CNC solution for STEP-compliant manufacturing." Robotics and Computer-Integrated Manufacturing 31 (2015): 1-10. https://doi.org/10.1016/i.rcim.2014.06.003

[9] T.M.S. Tg. Sulaiman, S.B. Mohamed, M. Minhat, A.S. Mo-hamed, and A.R. Mohamed., "Integrated Interface Development Environment using STEP Universal Data Structure." International Journal of Engineering \& Technology 7, no. 2.15 (2018): 27-29. https://doi.org/10.14419/ijet.v7i2.15.11194

[10] Mohamed, Saiful Bahri, Adnan Jameel, and Mohammad Minhat. "A review on intelligence STEP-NC data model and function blocks CNC machining protocol." In Advanced Materials Research, vol. 845, pp. 779-785. Trans Tech Publications Ltd, 2014.

https://doi.org/10.4028/www.scientific.net/AMR.845.779 
[11] Keong, Chen Wong, and Yusri Yusof. "A Design of New Product Database System for Supporting Step-Compliant Total Integration Applications." Journal of Advanced Research in Applied Mechanics 57, no. 1 (2019): 1-13.

[12] Kim, Byung Chul, Duhwan Mun, Soonhung Han, and Michael J. Pratt. "A method to exchange procedurally represented 2D CAD model data using ISO 10303 STEP." Computer-Aided Design 43, no. 12 (2011): 1717-1728. https://doi.org/10.1016/i.cad.2011.07.006

[13] Kretz, Daniel, Tobias Teich, Joerg Militzer, and Tim Neumann. "Implementing ISO standard 10303 application protocol 224 for automated process planning." Robotics and Computer-Integrated Manufacturing 27, no. 4 (2011): 729-734.

https://doi.org/10.1016/i.rcim.2010.12.010

[14] Xiao, Wenlei, Lianyu Zheng, Ji Huan, and Pei Lei. "A complete CAD/CAM/CNC solution for STEP-compliant manufacturing." Robotics and Computer-Integrated Manufacturing 31 (2015): 1-10.

https://doi.org/10.1016/i.rcim.2014.06.003

[15] Rauch, Matthieu, Raphael Laguionie, Jean-Yves Hascoet, and Suk-Hwan Suh. "An advanced STEP-NC controller for intelligent machining processes." Robotics and Computer-Integrated Manufacturing 28, no. 3 (2012): 375-384.75384.

https://doi.org/10.1016/j.rcim.2011.11.001 